INPLASY

PROTOCOL

To cite: Roquette et al. The

Development of Sport

Expertise: An Umbrella Review

of Systematic Reviews and

Meta-Analyses. Inplasy

protocol 2020120034. doi:

10.37766/inplasy2020.12.0034

Received: 06 December 2020

Published: 06 December 2020

Corresponding author:

João Roquette

joao.roquette@estadio.ulisboa.pt

Author Affiliation:

Universidade de Lisboa

Support: No sources of funding.

Review Stage at time of this submission: Data analysis.

Conflicts of interest:

None.

\section{The Development of Sport Expertise: An Umbrella Review of Systematic Reviews and Meta-Analyses}

Roquette, J1; Sarmento, H2; Araújo, D³; Güllich, A4.

Review question / Objective: This paper synthesizes the findings of systematic reviews and meta-analyses already available about Expertise and Talent Identification and Development (TID) research in sport.

Condition being studied: The aim of this article was to perform a systematic review of the systematics reviews and meta-analyses published about the development of sport expertise, looking for a bird's eye view about the most frequently researched topics, calling for an updated accumulation of research to better understanding of the spread of summary effects, heterogeneity, hints of bias and quality of empirical findings.

INPLASY registration number: This protocol was registered with the International Platform of Registered Systematic Review and Meta-Analysis Protocols (INPLASY) on 06 December 2020 and was last updated on 06 December 2020 (registration number INPLASY2020120034).

\section{INTRODUCTION}

Review question / Objective: This paper synthesizes the findings of systematic reviews and meta-analyses already available about Expertise and Talent Identification and Development (TID) research in sport.
Rationale: The scientific study of sport expertise has grown significantly over recent years with important implications both for talent identification and development (TID) in sport. However, the literature is replete of inconsistencies, including the language and definitions used. Therefore, it has become critical to develop an umbrella review about the 
scientific evidences with translation and truly impact for sport practices and programs on expertise and talent development.

Condition being studied: The aim of this article was to perform a systematic review of the systematics reviews and metaanalyses published about the development of sport expertise, looking for a bird's eye view about the most frequently researched topics, calling for an updated accumulation of research to better understanding of the spread of summary effects, heterogeneity, hints of bias and quality of empirical findings.

\section{METHODS}

Search strategy: This systematic review of systematic reviews and meta-analyses of the available literature was conducted according to PRISMA (Preferred Reporting Items for Systematic Reviews and Metaanalyses) guidelines. The electronic databases Web of ScienceTM Core Collection, Scopus and SPORTDiscus were searched for relevant publications to identify systematic reviews and metaanalyses about expertise and expert performance in sport, including talent identification and development, by using combination of the following keywords (Review OR Meta-analysis) AND (Talent* OR Expert* OR "Sport Expertise"). We also scanned the reference lists of these publications in order to find other important articles for this overview review. The publications included met the following criteria: (1) contained relevant data concerning expertise and expert performance in sport, including talent identification and/or development in sport; (2) be a systematic review or a metaanalysis and (3) were written in English language. Studies were excluded if they: (1) Did not contain any relevant data about expertise, expert performance and talent identification and development in sport and/or (2) are conference abstracts or book chapters. Two reviewers (JR, HS) independently screened citations and abstracts to identify articles potentially meeting the inclusion criteria. For those articles, full text versions were retrieved and independently screened by those reviewers, to determine whether they met inclusion criteria. Any disagreement regarding study eligibility was resolved in discussions including the other two reviewers (DA, AG).

Participant or population: Athletes from various sports and multiple levels of sport expertise.

Intervention: Not applicable.

Comparator: Not applicable.

Study designs to be included: Various study designs (cross-sectional, retrospective, longitudinal,...)

Eligibility criteria: The publications included met the following criteria: (1) contained relevant data concerning expertise and expert performance in sport, including talent identification and/or development in sport; (2) be a systematic review or a metaanalysis and (3) were written in English language.

Information sources: The electronic databases Web of ScienceTM Core Collection, Scopus and SPORTDiscus were searched for relevant publications. We also scanned the reference lists of these publications in order to find other important articles for this overview review.

Main outcome(s): This umbrella review highlights the difficulties of $\mathrm{Tl}$ in sport given the nonlinear nature of expertise and expert performance development and recognized important inconsistencies in the definition of some concepts used within sport expertise literature. Evidences detected in the reviewed studies about the importance of athlete-environment relationship, suggest that future research about the development of sport expertise need a multidimensional research. The results of empirical studies indicated that elite or expert athletes developed superior perceptual-cognitive skills which means a better perceptual performance. Moreover, studies examining developmental 
participation patterns of elite athletes revealed diversified engagement in different sports and non-organized sports during childhood and adolescence with practical implications for long-term sustainable athletic development and expert performance.

Data management: To organise the results, the studies were classified into categories established according to the major research topics that emerged from the content analysis.

Quality assessment / Risk of bias analysis: The quality of selected studies each were assessed using the AMSTAR 2, a critical appraisal tool for systematic reviews that included randomized or non-randomized studies of health care interventions, or both.

Strategy of data synthesis: The most common themes of analysis were grouped into four (4) overarching categories: (1) Performance structure; (2) Talent identification (TI) in sport; (3) Talent development (TD) in sport and (4) Methodology, terminology and taxonomy.

Subgroup analysis: The four (4) main categories were subdivided into subcategories: (1) Performance structure; i) Task constraints; ii) Performer's constraints (2) Talent identification (TI) in sport; i) Coach assessment; ii) Test development for measuring talent predictors; iii) Relative age effect (3) Talent development (TD) in sport; i) Performance trajectories; ii) Task constraints; iii) Performers' s constraints; iv) Environmental constraints; and (3) Methodology, terminology and taxonomy.

Sensibility analysis: Not applicable.

Language: The selection was for the original articles in English.

Country(ies) involved: Portugal and Germany.

Keywords: Sport Expertise; Talent Identification; Talent Development.
Contributions of each author:

Author 1 - João Roquette.

Email: joao.roquette@estadio.ulisboa.pt

Author 2 - Hugo Sarmento.

Email: hugo.sarmento@uc.pt

Author 3 - Duarte Araújo.

Email: daraujo@fmh.ulisboa.pt

Author 4 - Arne Güllich.

Email: guellich@sowi.uni-kl.de 